\title{
Mean-Variance efficient strategies in proportional reinsurance under group correlation in a gaussian framework
}

\author{
Flavio Pressacco ${ }^{1}$, Paolo Serafini ${ }^{2}$ and Laura Ziani $^{1}$
}

\begin{abstract}
The paper concerns optimal mean-variance proportional reinsurance under group correlation. In order to solve the corresponding constrained quadratic optimization problem, we make large recourse both to the smart friendly technique originally proposed by B. de Finetti in his pioneering paper and to the well known Karush-Kuhn-Tucker conditions for constrained optimization. We offer closed form results and insightful considerations about the problem. In detail, we give closed form formulae to express the efficient meanvariance retention set both in the retention space and in the mean-variance one.
\end{abstract}

Keywords. Mean-variance efficiency; constrained quadratic optimization; proportional reinsurance; group correlation.

Acknowledgement. We acknowledge financial support of MedioCredito Friuli Venezia Giulia through the "Bonaldo Stringher" Laboratory of Finance, Department of Finance, University of Udine.

\section{Introduction}

Proportional reinsurance has played an important historical role in the theory of decisions. Indeed, it was with reference to a proportional reinsurance problem that B. de Finetti introduced in his seminal paper [9] the mean-variance efficiency approach in modern financial decisions under uncertainty. This should be seen as the forerunner of the reward-risk analysis which plays a central role in the risk management of financial institutions (banks, insurance companies, pension funds, investment funds and so on).

Undoubtedly this and other works ([10], [11]) by de Finetti, concerning reinsurance arrangements, affected the thoughts of relevant actuarial researchers, see e.g. Borch [5], who in his milestone book, paid a lot of tributes to de Finetti's ideas about insurance issues and Gerber, who also in many papers ([13], [15], and jointly with Bühlmann [8]) recalled de Finetti's anticipatory thoughts in reinsurance arrangements. But, on the specific point of mean-variance optimal reinsurance choices of a single company, the application areas have been restricted to the case of no correlated risks. The issue has been treated e.g. by Bühlmann ([6], [7]) who was well aware of de Finetti's treatment in the no correlation case; Dickson and Waters [12] who, always with reference to the no correlation case, treated the issue of minimizing the ruin probability; Glineur and Walhin [17] who gave rigorous proof in terms of Kuhn-Tucker conditions [20] of de Finetti's results under no correlation; Lampaert and Walhin [21], who compared different type of reinsurance strategies including proportional reinsurance.

\footnotetext{
${ }^{1}$ Dipartimento di Finanza, Università di Udine

${ }^{2}$ Dipartimento di Matematica e Informatica, Università di Udine
} 
The reason of this attitude is probably due to the widespread opinion that closed form formulae of the whole set of proportional efficient retentions may be obtained only under no correlation, while in the correlation case there is the need to make recourse to a sequential procedure which is the counterpart of the critical line algorithm of the classical portfolio problem (see Markowitz [23], [24], [25]). The issue has been treated e.g. by Barone [3] who analyzed a specific point of the controversial issue raised by H. Markowitz [26] about the applicability of de Finetti's approach to the case of correlated risks and by Pressacco-Serafini [29].

While this statement is true in general, there is at least one specific structure of correlation which allows to recover nice results with closed form formulae. This special structure was named group correlation by de Finetti, who treated the point in his paper and gave a quick hint to some key properties of the efficient set (see [9], p. 27-30).

At our knowledge, the only subsequent treatment of this issue may be found in a paper by Gigante [16]. Recognizing the strict connection between the proportional reinsurance problem and the portfolio selection problem, early signalled by Pressacco [28], she applied the Karush-Kuhn-Tucker conditions ([19], [20]) to the constrained quadratic optimization problem arising from the mean-variance approach to proportional reinsurance. This way she was able to show that, under group correlation, a fast version of the critical line algorithm could be implemented to obtain the whole mean-variance set of efficient retentions. Anyway, she also did not get closed form formulae of the retentions.

In this paper, we fill this gap and offer a complete closed form solution of the proportional reinsurance problem under group correlation, jointly with an insightful economic interpretation of the efficient set. To explain our results, we make large recourse to de Finetti's original approach, based, as shown in Pressacco and Serafini [29], on the properties of the so called advantage functions.

In detail:

a. we show (Theorem 2 and Corollary 1) that there is a one to one correspondence between points of the mean-variance efficient set and a closed interval of values of a shadow price parameter, which reveals to be nothing but the counterpart of the set of optimal values of the Lagrange multiplier of the expectation constraint in the quadratic optimization approach;

b. we provide (formulae (13) and (14)) closed form formulae of the efficient retention vectors as a function of the shadow price parameter;

c. we show (Property 5) that in the $n$-dimensional space of retentions, the mean-variance efficient set is piecewise linear and continuous at corner points;

d. we specify (formula (12)) the values of the shadow price and hence of the retention vector corresponding to the corner points of the efficient set;

e. we explain (section 4) the economic meaning of such corners;

f. we show (formula (34)) that in the mean-variance space, the set of efficient retentions is a piecewise, continuous and without kinks union of parabolas, whose explicit equations are given.

We are well aware of the fact that, for non gaussian worlds, alternative risk measures are used in order to find mean-risk efficient sets in financial and reinsurance models ${ }^{1}$ and hope that our results about group correlation in a gaussian world may be useful for comparisons with those coming from modern mean-risk alternative models.

\footnotetext{
${ }^{1}$ Indeed, starting from the pioneer paper by Artzner et al. [1] on coherent risk measures, recent literature is focusing on some new approaches about risk measurement and is addressing to the topic of finding the proper corresponding measure of risk. See for instance and among others, Landsman and Sherris [22]; Rockafellar et al. [30]; Goovaerts et al. [18]; Miller and Ruszczynski [27]; Balbás et al. [2].
} 
The plan of the paper is as follows: in section 2 the basic results of the mean-variance efficiency are reported. In section 3 we describe the preliminary properties of proportional reinsurance under group correlation (3.1) and the structure of the mean-variance efficient retentions (3.2). Section 4 is devoted to a geometric view and economic meaning of the efficient path in the space of retentions. In section 5 we derive explicit formulae of the parabolic efficient set in mean-variance space. In section 6 , with reference to a textbook example, we offer a quick analysis of the influence of correlation on the concavity of the efficient mean-variance frontier and the related utility of proportional reinsurance in the riskiness control. Conclusions follow in section 7. Some proofs are presented in appendixes.

\section{Mean-variance efficiency in proportional reinsurance: basic re- sults}

Let us briefly recall the essentials of a proportional reinsurance problem. An insurance company is faced with $n$ risks (policies). The net profit, that is the difference between net premiums and losses, of these risks is described by a vector of random variables with expected values $\mathbf{m}>0$, and a non-singular covariance matrix $C$. We denote the $(i, j)$ entry of the matrix $C$ as $\sigma_{i j}$. The diagonal elements are denoted also as $\sigma_{i}^{2}=\sigma_{i i}$, and the $(i, j)$ entry may be alternatively denoted as $\sigma_{i j}=\rho_{i j} \sigma_{i} \sigma_{j}$ with $\rho_{i j}$ the correlation coefficient.

The company has to choose a proportional reinsurance or retention strategy specified by a retention vector $\mathbf{x}$. The retention is feasible if $0 \leq \mathbf{x} \leq 1$. By applying reinsurance on original terms, a retention $\mathbf{x}$ induces a random profit with expectation $E=\mathbf{x}^{\top} \mathbf{m}$ and variance $V(x)=\mathbf{x}^{\top} C \mathbf{x}$. How to choose $\mathbf{x}$ ?

In his seminal paper de Finetti [9] introduced the mean-variance paradigm in financial decisions under uncertainty suggesting that the choice should be restricted to the set of mean-variance efficient retentions, that is among those feasible $\mathbf{x}$ such that there are no feasible retentions $\mathbf{y}$ with $E(\mathbf{y}) \geq E(\mathbf{x}), V(\mathbf{y}) \leq V(\mathbf{x})$ and at least one of the two inequalities holding in a strict sense.

Keeping account of the condition $\mathbf{m}>0$, which implies that feasible values for $E$ are those of the closed interval $\left[0, \mathbf{1}^{\top} \mathbf{m}\right]$, the efficient set is found solving for any $E$ of that interval the constrained optimization problem:

$$
\begin{aligned}
\min & \frac{1}{2} \mathbf{x}^{\top} C \mathbf{x} \\
& \mathbf{x}^{\top} \mathbf{m} \geq E \\
& \mathbf{0} \leq \mathbf{x} \leq \mathbf{1}
\end{aligned}
$$

A standard way to solve the problem is to introduce the Lagrangian:

$$
L(\mathbf{x}, \lambda, \mathbf{u}, \mathbf{v})=\frac{1}{2} \mathbf{x}^{T} C \mathbf{x}+\lambda\left(E-\mathbf{m}^{T} \mathbf{x}\right)+\mathbf{u}(\mathbf{x}-\mathbf{1})-\mathbf{v} \mathbf{x}
$$

and make recourse to the KKT conditions which state that $\hat{\mathbf{x}}$ is optimal iff there exists a triple $(\hat{\lambda}, \hat{\mathbf{u}}, \hat{\mathbf{v}}), \lambda \geq 0$, $\hat{\mathbf{u}} \geq \mathbf{0}, \hat{\mathbf{v}} \geq \mathbf{0}$ such that:

1) $\hat{\mathbf{x}}$ minimizes $L(\mathbf{x}, \hat{\lambda}, \hat{\mathbf{u}}, \hat{\mathbf{v}})$

2) $\hat{\mathbf{x}}$ is feasible

3) either $\hat{x}_{j}=0$ or $\hat{v}_{j}=0$ (or both) and either $\hat{x}_{j}=1$ or $\hat{u}_{j}=0$ (or both)

It is rather surprising to find that the optimality conditions may be eventually expressed in a very simple way through the functions:

$$
F_{i}(\mathbf{x})=\frac{1}{2} \frac{\frac{\partial V}{\partial x_{i}}}{\frac{\partial E}{\partial x_{i}}}:=\sum_{j=1}^{n} \frac{\sigma_{i j}}{m_{i}} x_{j} \quad i=1, \ldots, n
$$


introduced by de Finetti as a tool to find, through an intuitive simple procedure, the mean-variance efficient set at a time where the KKT conditions were not yet available. In a previous paper we propose to call these functions advantage functions, as they intuitively capture the advantage coming at a retention point $\mathbf{x}$ from a marginal (additional or initial) reinsurance of the $i$-th risk. The advantage is measured precisely by the ratio (one half) decrease of variance over decrease of expectation. Then, the efficient set can be simply characterized in terms of the advantage functions as follows (for details see [29], p. 33):

Optimality condition: $\hat{\mathbf{x}}$ is mean-variance efficient iff there exists $\lambda \geq 0$ such that:

$$
\begin{array}{rll}
\text { i) } & F_{i}(\hat{\mathbf{x}})=\lambda & \text { if } 0<\hat{x}_{i}<1 \\
\text { ii) } & F_{i}(\hat{\mathbf{x}}) \geq \lambda & \text { if } \hat{x}_{i}=0 \\
\text { iii) } & F_{i}(\hat{\mathbf{x}}) \leq \lambda & \text { if } \hat{x}_{i}=1
\end{array}
$$

The intuitive meaning of the condition is clear if we look at the advantage function $F_{i}(\mathbf{x})$ as the pseudo marginal utility at $\mathbf{x}$ of buying reinsurance of the $i$-th risk and at $\lambda$ as the shadow price of any (marginal in quota terms) reinsurance. In particular, the optimality condition means that, given the shadow price, we buy reinsurance if the marginal utility is larger than the price and stop buying it at the point where the (diminishing) marginal utility just matches the price, or obviously if zero retention has been reached this way.

As said in the introduction, even in the absence of the KKT conditions, de Finetti was able to use the properties of the advantage functions to find a closed form solution for the mean-variance efficient set in case of no correlation, that is $\sigma_{i j}=0$. Precisely, he obtained (see [9], p. 10):

$$
\hat{x}_{i}(\lambda)=\min \left\{\lambda \frac{m_{i}}{\sigma_{i}^{2}}, 1\right\} \quad i=1, \ldots, n ; \quad \lambda \geq 0
$$

He correctly argued that in the more general case of correlation, there was no hope to obtain closed form formulae, but observed that some "regularity" properties could help in the analysis of a special structure of correlation which he named group correlation.

As said in the introduction, fifty years later Gigante [16] applied the KKT conditions to the constrained quadratic optimization problem arising from the application of the mean-variance approach to proportional reinsurance under group correlation. This way, she obtained a fast version of the critical line algorithm to get, through a sequential procedure (and not in closed form), the whole set of the mean-variance efficient retentions.

In the next section we will see how to exploit the optimality condition to find closed form solutions for the set of efficient retentions under group correlation.

\section{Proportional reinsurance under group correlation}

\subsection{Preliminary}

According to de Finetti's definitions ([9], p. 27), group correlation means that the set of insured risks may be partitioned into a number $g$ of groups characterized by the following properties:

a. the correlation between each pair of policies belonging to the same group $q, q=1, \ldots, g$ (shortly the correlation within) is a fixed group specific positive constant $\rho_{q}>0$, whereas the correlation between pairs of risks of different groups (shortly the correlation between) is identically zero;

b. the ratio between standard deviation and expectation of the random gain of any policy is a group specific constant ratio $\frac{\sigma_{i, q}}{m_{i, q}}=a_{q}$ for any risk $i$ of the group $q$. In technical terms, this amounts to say that 
the insurance premiums are charged by a safety loading based on a standard deviation principle (see for example Berliner, [4] and Gerber [14]), with a loading coefficient common to all risks within the same group.

Let us now introduce and explain some notations.

- $x_{i, q}, i=1, \ldots, n_{q}$ is the individual retention of the $i$-th risk in group $q$

- $\mathbf{x}_{q}, q=1, \ldots, g$ is the vector of retentions of the group $q$. In particular $\mathbf{1}_{q}$ (respectively $\mathbf{0}_{q}$ ) is the vector of full (zero) retentions for all risks of the group $q$

- $\mathrm{x}$ is the vector of global retentions of the risks of all groups

- $F_{i, q}(\mathbf{x})$ is the value, at $\mathbf{x}$, of the advantage function of risk $i$ in group $q$

It is straightforward to check that, under group correlation, the advantage function of the $i$-th risk of the group $q$ becomes

$$
F_{i, q}(\mathbf{x})=a_{q}\left(x_{i, q} \sigma_{i, q}+\rho_{q} \sum_{j \neq i} x_{j, q} \sigma_{j, q}\right)
$$

which may be rewritten as follows:

$$
F_{i, q}(\mathbf{x})=a_{q}\left[x_{i, q} \sigma_{i, q}\left(1-\rho_{q}\right)+\rho_{q} \sum_{j=1}^{n_{q}} x_{j, q} \sigma_{j, q}\right]
$$

Remark 3.1. $F_{i, q}(\mathbf{x})=F_{i, q}\left(\mathbf{x}_{q}\right)$, owing to zero correlation between groups.

Remark 3.2. $F_{i, q}(\mathbf{x}) \geq 0 ; F_{i, q}(\mathbf{x})=0 \Leftrightarrow \mathbf{x}_{q}=\mathbf{0}_{q}$.

After that, the optimality condition under group correlation becomes:

Optimality condition under group correlation: $\hat{\mathbf{x}}$ is mean-variance efficient iff there exists $\lambda \geq 0$ such that, for any $q=1, \ldots, g$ :

$$
\begin{array}{lll}
\text { I) } & F_{i, q}(\hat{\mathbf{x}})=a_{q}\left(\hat{x}_{i, q} \sigma_{i, q}+\rho_{q} \sum_{j \neq i} \hat{x}_{j, q} \sigma_{j, q}\right)=\lambda & \text { if } 0<\hat{x}_{i, q}<1 \\
\text { II) } & F_{i, q}(\hat{\mathbf{x}})=a_{q} \rho_{q} \sum_{j \neq i} \hat{x}_{j, q} \sigma_{j, q} \geq \lambda & \text { if } \hat{x}_{i, q}=0 \\
\text { III }) & F_{i, q}(\hat{\mathbf{x}})=a_{q}\left(\sigma_{i, q}+\rho_{q} \sum_{j \neq i} \hat{x}_{j, q} \sigma_{j, q}\right) \leq \lambda & \text { if } \hat{x}_{i, q}=1
\end{array}
$$

In what follows, we will find convenient to order the risks within each group coherently with their standard deviation in decreasing order, that is use the labeling induced by:

$$
\sigma_{1, q}>\sigma_{2, q}>\ldots>\sigma_{n_{q}, q}
$$

Remark 3.3. Multiplying each standard deviation by the common coefficient $a_{q}$ does not alter the ordering, which then is the same induced by the ratio variance over expectation, which played a key role in the no correlation case ([29], par. 3).

In the same line of reasoning, an ordering among groups will be introduced according to the values at $\mathbf{x}=\mathbf{1}$ (or $\mathbf{x}_{q}=\mathbf{1}_{q}$ ) of the individual advantage functions of the first risk of each group. Coherently, we will label groups so as:

$$
F_{1,1}\left(\mathbf{1}_{1}\right)>F_{1,2}\left(\mathbf{1}_{2}\right)>\ldots>F_{1, g}\left(\mathbf{1}_{g}\right)
$$

After that, let us resume here some fundamental results about the structure of mean-variance efficient retentions (under group correlation). 
Result 1. $\mathbf{x}_{q}=\mathbf{0}_{q} \Rightarrow \lambda \leq 0$.

Indeed, Remark 3.2 and KKT II), applied to $x_{i, q}=0$, imply $\lambda \leq F_{i, q}(\mathbf{x})=F_{i, q}\left(\mathbf{0}_{q}\right)=0$.

Result 2. $x_{i, q}>0 \Rightarrow \lambda>0$.

Indeed, Remark 3.2 and KKT I) or III) applied to $x_{i, q}>0$, imply $0<F_{i, q}\left(\mathbf{x}_{q}\right)=F_{i q}(\mathbf{x}) \leq \lambda$.

Results 1 and 2 jointly imply:

Result 3. If there exists a $q$ s.t. $\mathbf{x}_{q}=\mathbf{0}_{q}$ then $\mathbf{x}=0$, or, equivalently, if there exists a pair $\{i, q\}$ s.t. $x_{i, q}>0$ then, for any $q^{\prime}$, there exists a pair $\left\{i^{\prime}, q^{\prime}\right\}$ s.t. $x_{i^{\prime}, q^{\prime}}>0$.

Verbally, this means that, if in a given group at least one retention is strictly positive, then there is a strictly positive retention in any group.

Result 4. $x_{i, q}>0 \Rightarrow x_{h, q}>0$, for any $h$.

Proof: Ad absurdum, let $x_{h, q}=0$, then $\left(F_{i, q}-F_{h, q}\right)=a_{q}\left(1-\rho_{q}\right) x_{i, q} \sigma_{i, q}>0$. On the other side, KKT imply that $F_{i, q} \leq \lambda \leq F_{h, q}$, that is $\left(F_{h, q}-F_{i, q}\right) \geq 0$, contradicting the previous inequality. $\diamond$

An immediate corollary of Results 3 and 4 is:

Property 1. In any efficient retention, either $\mathbf{x}=\mathbf{0}$ (and $\lambda=0$ ) or $\mathbf{x}>\mathbf{0}$ (and $\lambda>0$ ).

Verbally, either all null retentions or all strictly positive retentions.

Let us now concentrate on some properties of $\mathbf{x}_{q}$ vectors belonging to an efficient $\mathbf{x}$ vector.

Property 2. If $x_{i, q}=1$ then $x_{h, q}=1$ for all $h>i$ (remember the labeling induced by the order of $\sigma$ ).

Proof: Indeed, $i<h \Rightarrow \sigma_{i, q}>\sigma_{h, q} \geq x_{h, q} \sigma_{h, q} \Rightarrow\left(F_{i, q}-F_{h, q}\right)=a_{q}\left(1-\rho_{q}\right)\left(\sigma_{i, q}-x_{h, q} \sigma_{h, q}\right)>0$. Suppose ad absurdum $0<x_{h, q}<1$, then KKT imply $F_{i, q} \leq \lambda=F_{h, q}$, that is $\left(F_{i, q}-F_{h, q}\right) \leq 0$ contradicting the previous inequality.

Property 3. If $0<x_{i, q}, x_{h, q}<1$ then $x_{i, q} \sigma_{i, q}=x_{h, q} \sigma_{h, q}$.

Proof: Indeed, $\left(F_{i, q}-F_{h, q}\right)=a_{q}\left(1-\rho_{q}\right)\left(x_{i, q} \sigma_{i, q}-x_{h, q} \sigma_{h, q}\right)$. On the other side, by KKT, $F_{i, q}=F_{h, q}=\lambda$, that is $\left(F_{i, q}-F_{h, q}\right)=0$.

It is proper to signal that Properties 1, 2 and 3 have been discovered by de Finetti ([9], pg. 28-30) as an informal consequence of his dynamic approach, while 2 and 3 have been later rigorously proved by Gigante ([16], pg. 155, 156) making recourse to the KKT conditions, albeit without exploiting the advantage functions approach. Quite likely this is an explanation of her inability in reaching closed form formulae for efficient retentions.

On the contrary, we choose to follow a proper "integrated approach" between KKT conditions and de Finetti's advantage functions, obtaining this way original results which we will discuss in the next section. 


\subsection{The basic structure of the mean-variance efficient retentions}

Results and properties of the last section suggest the following Theorem:

Theorem 1. The structure of any group efficient retention $\hat{\mathbf{x}}_{q}$, which is part of an efficient retention $\hat{\mathbf{x}}$, is defined, given a feasible choice of $\hat{x}_{1, q}$, by:

$$
\hat{x}_{i, q}=\min \left\{\frac{\hat{x}_{1, q} \sigma_{1, q}}{\sigma_{i, q}} ; 1\right\} \quad i=1, \ldots, n_{q}
$$

Proof: If $\hat{x}_{1, q}<1$ and $\hat{x}_{i, q}<1$, the thesis is implied by Property 3 . If $\hat{x}_{1, q}<1$ and $\hat{x}_{i, q}=1$ the optimality condition yields $F_{1, q}(\hat{\mathbf{x}})=\lambda$ and $F_{i, q}(\hat{\mathbf{x}}) \leq \lambda$ so that

$$
a_{q}\left(\hat{x}_{i, q} \sigma_{i, q}\left(1-\rho_{q}\right)+\rho_{q} \sum_{j=1}^{n_{q}} \hat{x}_{j, q} \sigma_{j, q}\right) \leq a_{q}\left(\hat{x}_{1, q} \sigma_{1, q}\left(1-\rho_{q}\right)+\rho_{q} \sum_{j=1}^{n_{q}} \hat{x}_{j, q} \sigma_{j, q}\right)
$$

implying

$$
\frac{\hat{x}_{1, q} \sigma_{1, q}}{\sigma_{i, q}} \geq \hat{x}_{i, q}=1
$$

and the expression (6) is satisfied. If $\hat{x}_{1, q}=1$ and $\hat{x}_{i, q}=1$, the expression (6) is satisfied because of the ordering convention (4). The case $\hat{x}_{1, q}=1$ and $\hat{x}_{i, q}<1, i>1$, is ruled out by Property 2 .

Remark 3.4. The vector $\hat{\mathbf{x}}_{q}$ is univocally defined by $\hat{x}_{1, q}$. It is expressive to say that $\hat{x}_{1, q}$ is the "driver" of $\hat{\mathbf{x}}_{q}$.

Let $h$ be the smallest integer such that $\hat{x}_{h, q}=1$. If none exists, set $h:=n_{q}+1$. By Property $3, \hat{x}_{i, q}=1$ for $i \geq h$. Hence, by using (6), we may write, for $i=1, \ldots, h-1$ :

$$
F_{i, q}(\hat{\mathbf{x}})=F_{i, q}\left(\hat{\mathbf{x}}_{q}\right)=a_{q}\left(\hat{x}_{1, q} \sigma_{1, q}\left(1+\rho_{q}(h-2)\right)+\rho_{q} \sum_{j=h}^{n_{q}} \sigma_{j, q}\right)=\lambda
$$

Therefore, given $\hat{x}_{1, q}, F_{i, q}\left(\hat{\mathbf{x}}_{q}\right)$ is constant for any $i=1, \ldots, h-1$, the value of this constant being precisely $\lambda$ by the optimality conditions.

So we may write $\lambda\left(\hat{\mathbf{x}}_{q}\right)=\lambda\left(\hat{x}_{1, q}\right)$.

Let us now give a closer look at (7) in order to understand the connection between $\hat{x}_{1, q}$ and $\lambda$. It is clear that $\lambda$ may be thought as a function of $\hat{x}_{1, q}$ and $h$; in turn, $h$ is a group specific function of $\hat{x}_{1, q}$. Hence $\lambda$ is a composite function $\varphi$ of $\hat{x}_{1, q}$ and we may write

$$
\lambda=\varphi\left(\hat{x}_{1, q}\right):=a_{q}\left(\hat{x}_{1, q} \sigma_{1, q}\left(1+\rho_{q}\left(h\left(\hat{x}_{1, q}\right)-2\right)\right)+\rho_{q} \sum_{j=h}^{n_{q}} \sigma_{j, q}\right)
$$

From the definition of $h$ and from (6)

$$
h\left(\hat{x}_{1, q}\right):=\min \left\{i: \hat{x}_{i, q}=1\right\}=\min \left\{i: \hat{x}_{1, q} \sigma_{1, q} \geq \sigma_{i, q}\right\}
$$

According to (9) and the ordering $(4), h\left(\hat{x}_{1, q}\right)$ is a lower semi-continuous decreasing step function with $n$ down jumps of height equal to 1 at the points $\hat{x}_{1, q}=\sigma_{h, q} / \sigma_{1, q}=: \hat{x}_{1, q}^{h}$ for $h=n_{q}, \ldots, 1$ and with $h(0)=n_{q}+1$ and $h(1)=1$, where the last jump takes place (see Figure 1). 


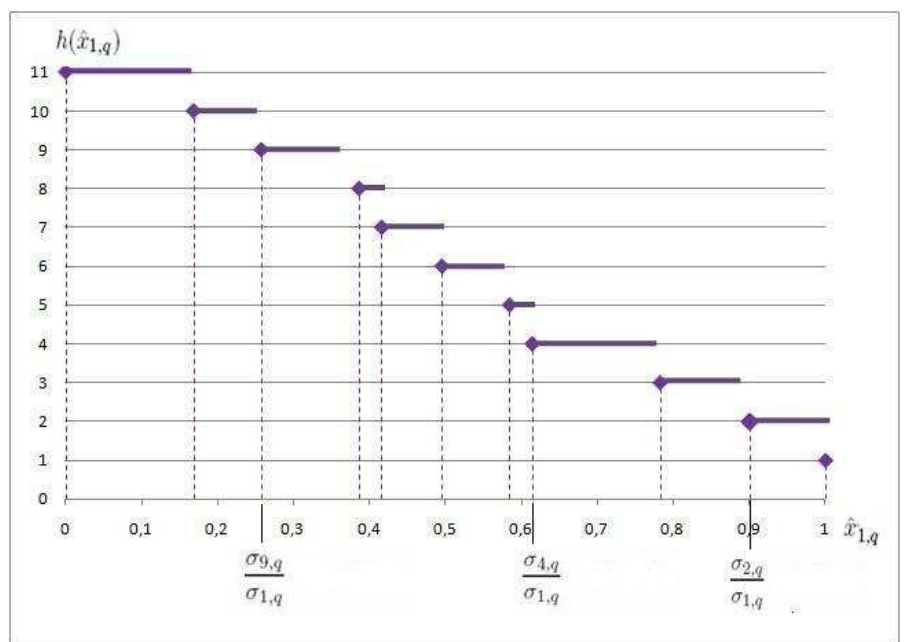

Figure 1: An example of the function $h\left(\hat{x}_{1, q}\right)$ with $n_{q}=10$ policies.

Then the properties of the function $\varphi\left(\hat{x}_{1, q}\right)$ (shortly denoted also as $\varphi_{q}$ ) can be summarized by:

Property 4. $\varphi\left(\hat{x}_{1, q}\right)$ is piecewise linear concave increasing function on the whole interval $[0,1]$. In particular, $\varphi_{q}(0)=0$ and $\varphi_{q}(1)=a_{q}\left(\sigma_{1, q}+\rho_{q} \sum_{j=2}^{n_{q}} \sigma_{j, q}\right)$, henceforth denoted by $\lambda_{q}$, which is the maximum of $\varphi_{q}$ on $[0,1]$.

In particular, for $q=1$

$$
\lambda=\varphi\left(\hat{x}_{1,1}\right)=\varphi_{1}=a_{1}\left(\hat{x}_{1,1} \sigma_{1,1}\left(1+\rho_{1}(h-2)\right)+\rho_{1} \sum_{j=h}^{n_{1}} \sigma_{j, 1}\right)
$$

See in Figure 2 the function $\varphi\left(\hat{x}_{1, q}\right)$ for the same parameters as in Figure 1.

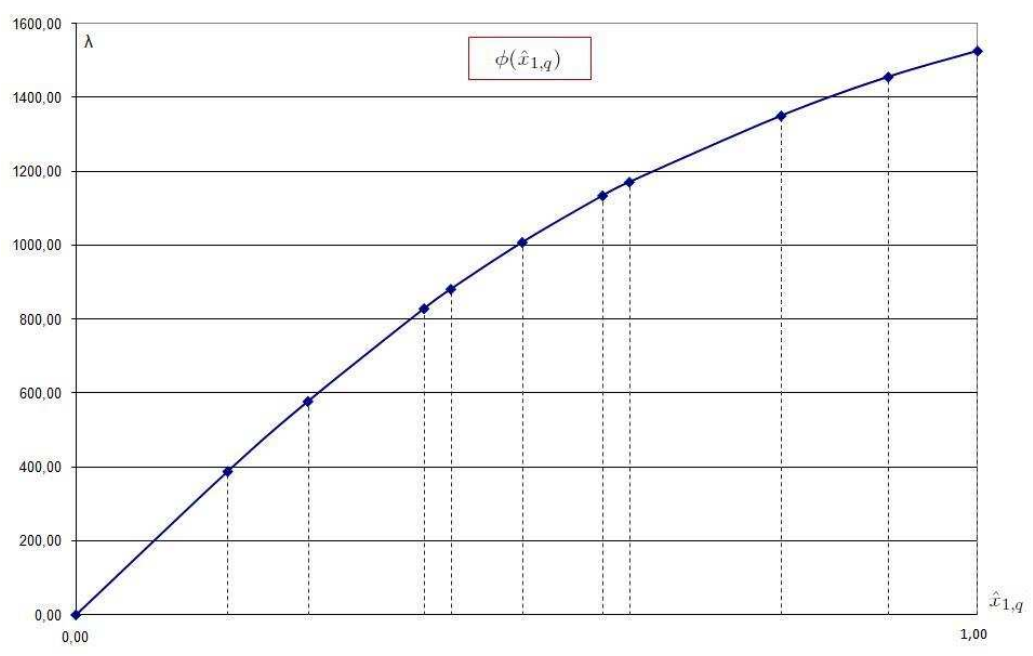

Figure 2: An example of the function $\varphi\left(\hat{x}_{1, q}\right)$.

Remark 3.5. Property 4 grants that $\varphi_{q}(\lambda)$ may be inverted on the interval $\left(0 \leq \lambda \leq \lambda_{q}\right)$ with a piecewise linear convex increasing inverse. 
The previous results allow a simple and powerful characterization of the set of all global efficient retentions $\hat{\mathbf{x}}$ via the functions $\varphi_{q}$, as shown in the following theorem.

Theorem 2. For any given $\lambda \geq 0$ a global efficient retention $\hat{\mathbf{x}}$ is given by the collection of the group efficient retentions $\hat{\mathbf{x}}_{q}$ obtained applying (6) to a set $\hat{x}_{1, q}$ of "drivers" given by:

$$
\hat{x}_{1, q}=\varphi_{q}^{-1}(\lambda) \quad \text { if } \quad 0 \leq \lambda \leq \lambda_{q} ; \quad \hat{x}_{1, q}=1 \quad \text { if } \quad \lambda>\lambda_{q}
$$

Remark 3.6. As $\lambda_{1}$ is, by the labeling (5), the largest $\lambda_{q}$, we may restrict $\lambda$ to the interval $\left[0, \lambda_{1}\right]$.

Corollary 1. There is a one-to-one correspondence between values of the shadow price $\lambda$ in the interval $\left[0, \lambda_{1}\right]$ and the set $\hat{\mathbf{x}}(\lambda)$ of efficient retentions.

As for the operational technique to find the drivers $\hat{x}_{1, q}$ of $\hat{\mathbf{x}}(\lambda)$, a two-step procedure is needed.

1. First step: find $h=h(q, \lambda)$ such that at $\lambda$ exactly $(h-1)$ risks have $\hat{x}_{i, q}<1$ or $h$ is the smallest integer $i$ such that $\hat{x}_{i, q}(\lambda)=1$.

Concretely, compute for $k=1, \ldots, n_{q}$ the sequence of "critical values" $\lambda\left(\hat{x}_{1, q}^{k}\right)$; these are the values of the shadow price at which the $k$-th risk of the group $q$ enters in reinsurance; the sequence is given by:

$$
\varphi\left(\hat{x}_{1, q}^{k}\right)=\lambda\left(\hat{x}_{1, q}^{k}\right)=F_{1, q}\left(\hat{x}_{1, q}^{k}\right)=F_{1, q}\left(\hat{\mathbf{x}}_{q}^{k}\right)=a_{q}\left(\sigma_{k, q}\left(1+\rho_{q}(k-2)\right)+\rho_{q} \sum_{j=k}^{n_{q}} \sigma_{j, q}\right)
$$

For the sake of completeness, let us put:

$$
\lambda\left(\hat{x}_{1, q}^{n+1}\right)=0 \quad \text { and } \quad \lambda\left(\hat{x}_{1, q}^{0}\right)=+\infty
$$

Then, choose $h:=k$ such that $\varphi\left(\hat{x}_{1, q}^{k}\right)=\lambda\left(\hat{x}_{1, q}^{k}\right) \leq \lambda<\lambda\left(\hat{x}_{1, q}^{k-1}\right)$.

It is clear that the critical values of $\lambda$ correspond to the jump points of $h\left(\hat{x}_{1, q}\right)$. At those points $\varphi\left(\hat{x}_{1, q}\right)$ is not differentiable but still continuous (see Figure 2).

2. Second step: insert $h$ in equation (8) and solve for $\hat{x}_{1, q}(\lambda)$ obtaining:

$$
\hat{x}_{1, q}(\lambda)=\varphi_{q}^{-1}(\lambda)=\frac{\lambda}{a_{q} \sigma_{1, q}\left(1+\rho_{q}(h-2)\right)}-\rho_{q} \frac{\sum_{j=h}^{n_{q}} \sigma_{j, q}}{\sigma_{1, q}\left(1+\rho_{q}(h-2)\right)}
$$

This formula gives the driver $\hat{x}_{1, q}$ and hence the whole vector $\hat{\mathbf{x}}_{q}$ as a linear function of $\lambda$. As for the other policies, we have:

$$
\begin{aligned}
& \hat{x}_{i, q}(\lambda)=\frac{\lambda}{a_{q} \sigma_{i, q}\left(1+\rho_{q}(h-2)\right)}-\rho_{q} \frac{\sum_{j=h}^{n_{q}} \sigma_{j, q}}{\sigma_{i, q}\left(1+\rho_{q}(h-2)\right)} \quad i=1, \ldots,(h-1) \\
& \hat{x}_{i, q}(\lambda)=1 \quad i=h, \ldots, n_{q}
\end{aligned}
$$

With obvious meaning of the symbols we could write:

$$
\begin{aligned}
& \hat{x}_{i, q}(\lambda)=A_{i, q} \lambda+B_{i, q} \quad i=1, \ldots,(h-1) \\
& \hat{x}_{i, q}(\lambda)=1 \quad i=h, \ldots, n_{q}
\end{aligned}
$$

Finally, repeat the procedure for any $q$.

Remark 3.7. The whole efficient set $\hat{\mathbf{x}}$ is in one-to-one correspondence with the interval $[0,1]$ of feasible retentions $\hat{x}_{1,1}$ of the first risk of the first group. Indeed, given the choice of $x_{1,1}$, we obtain $\lambda=\varphi\left(\hat{x}_{1,1}\right)$ from (10), the set of drivers $\hat{x}_{1, q}$ by (11) and finally the whole set by (6). 
We underline that the above expressions (13) and (14) incorporate a dependence on $h$, that is they hold, for each group $q$, only for values of $\lambda\left(\hat{x}_{1, q}^{h}\right) \leq \lambda<\lambda\left(\hat{x}_{1, q}^{h-1}\right)$. The following property holds:

Property 5. On the plane shadow price-retentions, the retentions are continuous piecewise linear functions of $\lambda$. They have common corner points at the group $q$ critical values of the shadow price (see Figure 3 as an example). The continuity at the corner points follows from Remark 3.5.

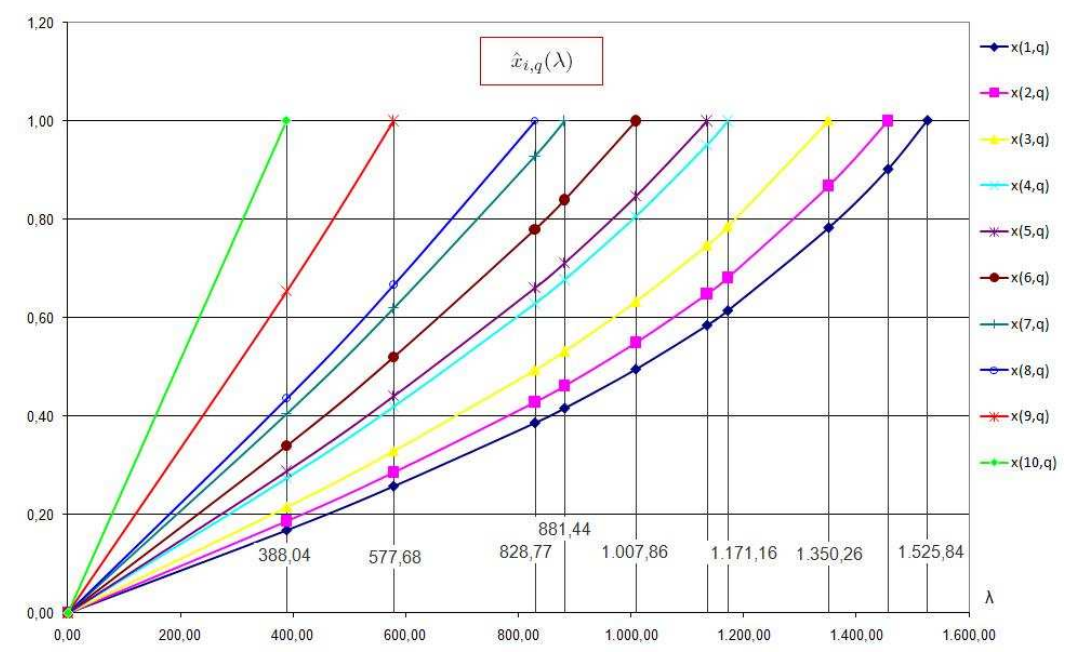

Figure 3: An example of the function $\hat{x}_{i, q}(\lambda)$.

Keeping in consideration different groups, the intervals of linearity do not coincide anymore. But, if we denote by $\left\{I_{q}\right\}$ the set of $\lambda$ intervals of the group $q$, joint linearity is recovered for all risks in the new set of intervals obtained by the intersection $\bigcap_{q}\left\{I_{q}\right\}$ of such a family of intervals. Or, said another way, if we consider the union over all groups of the critical values of $\lambda$, each retention quota (of all risks of all groups) is, in any interval between two consecutive critical values of $\lambda$, a linear function of $\lambda$. And on the plane shadow price-retentions, all the retentions are continuous piecewise linear functions with common corner points at the union of critical values of the shadow price.

\section{A geometric look at the efficient path in the space of retentions}

In order to understand the geometric properties of the efficient set and its economic meaning, let us consider the $n$ dimensional unit cube of feasible retentions. According to de Finetti's intuition, the efficient mean-variance set is a path in this cube. It should connect the natural starting point, the vertex $\mathbf{1}$ of full retention (with the largest expectation) to the opposite vertex $\mathbf{0}$ of full reinsurance (zero retention and hence minimum null variance). We found previously that there is a one to one correspondence between points of the efficient set (path) and points of the shadow price in the closed interval $\left(0, \lambda_{1}\right)$. Of course, $\lambda_{1}$ corresponds to the vertex of global full retention, where the value of the advantage function of the first risk of the first group matches that value of the shadow price, and $\mathbf{0}$ to the vertex of null retention. Each interval of values of the shadow price between two consecutive values of the global critical set corresponds to a segment of the path; there, all retentions are linear functions of $\lambda$. Keeping account of the continuity of each retention as a function of the shadow price, it turns out that the representation of the efficient set in the retention space is piecewise linear with corner points corresponding to the global set of critical values of the shadow price. 
At each corner point a risk previously fully retained begins to be reinsured. There its advantage function matches from below the (diminishing) value of the shadow price, matching at the same time the advantage function of all risks already partially reinsured. The value of the advantage function of all other risks, still fully retained, is lower than the current shadow price.

In each segment joining two adjacent corner points the set of partially reinsured risks does not change. Simply their retentions regularly (linearly) decrease in a way preserving, within each group, the equality of the products $\hat{x}_{i, q} \sigma_{i, q}$; in addition, the equality of all advantage functions of the set of reinsured risks of all groups is preserved at the shadow price level. At the end point (big crunch) of the path, all advantage functions jointly reach the smallest value 0 of the shadow price.

\section{The efficient set in the mean-variance space}

\subsection{Global expected return of the portfolio}

In the modern portfolio theory, when expected return is plotted against variance the efficient frontier is an union of parabolas (see [25], Theorem 7.12, p. 176). Points along this line represent optimal portfolios for which there is lowest risk for any given level of expected return.

In our context, how is the efficient frontier made? Intuitively, it will be still a sequence of pieces of parabolas connecting each other with continuity and derivability, as we will show hereinafter. Let us demonstrate this claim.

Let us consider the overall expected return of the generic group $q$, in each interval where there are $(h-1)$ active policies of the group; it is a function of $\lambda$ :

$$
E_{q}(\lambda)=\sum_{i=1}^{h-1} \hat{x}_{i, q}(\lambda) m_{i, q}+\sum_{i=h}^{n_{q}} m_{i, q}
$$

with $\hat{x}_{i, q}(\lambda)$ given by the (14a). Taking into account that $\frac{m_{i, q}}{\sigma_{i, q}}=\frac{1}{a_{q}}$, we obtain:

$$
\begin{aligned}
E_{q}(\lambda) & =\sum_{i=1}^{h-1}\left[\frac{\lambda}{a_{q}^{2}\left[1+\rho_{q}(h-2)\right]}-\rho_{q} \frac{\sum_{j=h}^{n_{q}} \sigma_{j, q}}{a_{q}\left[1+\rho_{q}(h-2)\right]}\right]+\sum_{i=h}^{n_{q}} m_{i, q} \\
& =\lambda\left[\frac{(h-1)}{a_{q}^{2}\left[1+\rho_{q}(h-2)\right]}\right]-\rho_{q} \frac{(h-1) \sum_{j=h}^{n_{q}} \sigma_{j, q}}{a_{q}\left[1+\rho_{q}(h-2)\right]}+\sum_{i=h}^{n_{q}} m_{i, q}
\end{aligned}
$$

which again could be well-rewritten in a more expressive way:

$$
E_{q}(\lambda)=\lambda \alpha_{q, h}+\beta_{q, h}
$$

as a linear function of the shadow price $\lambda$. Note that both:

$$
\begin{gathered}
\alpha_{q, h}=\frac{(h-1)}{a_{q}^{2}\left[1+\rho_{q}(h-2)\right]} \\
\beta_{q, h}=-\frac{\rho_{q}(h-1) \sum_{j=h}^{n_{q}} \sigma_{j, q}}{a_{q}\left[1+\rho_{q}(h-2)\right]}+\sum_{i=h}^{n_{q}} m_{i, q}
\end{gathered}
$$

are group and interval specific coefficients, through the dependence on the $\left(a_{q}, \rho_{q}\right)$ parameters couple and, of course, in the specific interval, also on $h_{q}$. Not surprisingly, the slope $\alpha_{q, h}$ is positive for any $h=2, \ldots, n_{q}+1$.

Now, in order to consider the global expected return of the entire portfolio, $E(\lambda)$, coming from all groups $g$, we need the remark and considerations at the end of the previous section 3.2. 
Precisely, on a specific subinterval of $\lambda$, where each group has a group specific number $(h-1)_{q}$ of active policies, the global expected return is given simply by the sum of the expectations of each of them.

In other terms, keeping in consideration all groups, there is still a subinterval specific linear relation between the global expectation and the value of the shadow price $\lambda$ :

$$
E(\lambda)=\lambda \alpha_{h}+\beta_{h}
$$

where, of course:

$$
\begin{aligned}
\alpha_{h} & =\sum_{q=1}^{g} \alpha_{q, h} \\
\beta_{h} & =\sum_{q=1}^{g} \beta_{q, h}
\end{aligned}
$$

We stress again that the coefficients $\alpha_{h}$ and $\beta_{h}$ are interval specific. In detail:

a. the slope $\alpha_{h}$ is increasing with $h$

Proof: First of all note that, moving from $h$ to $h+1$ determines a change only in one of the $\alpha_{q, k}$ coefficients, corresponding to the group whose the new risk entering in reinsurance is member. With reference to this group, consider $\alpha_{q, h+1}$ and $\alpha_{q, h}$ in order to show that $\alpha_{q, h+1}>\alpha_{q, h}$, or, keeping account of formula (19), that:

$$
\frac{h}{a_{q}^{2}\left(1+\rho_{q}(h-1)\right)}>\frac{(h-1)}{a_{q}^{2}\left(1+\rho_{q}(h-2)\right)}
$$

immediately verified for $h=1$ and easily verified, by elementary algebra, for $h \geq 2$.

b. the role of $\beta_{h}$ is to grant continuity at the connection points. In turn, the continuity of

$$
E_{q}(\lambda)=\sum_{i=1}^{n_{q}} m_{i, q} \hat{x}_{i, q}(\lambda)
$$

comes immediately from the continuity of $\hat{x}_{i, q}(\lambda)$. See Property 5.

From (21) we get also the inverse, still interval specific, relation:

$$
\lambda=\frac{E-\beta_{h}}{\alpha_{h}}
$$

which will be helpful hereinafter.

\subsection{Global variance of the portfolio}

With regard to the variance of the generic group $q$, we should have to deal with a potentially huge covariance matrix:

$$
V=\mathbf{x}^{\top} C \mathbf{x}=\sum_{i} \sum_{j} \hat{x}_{i, q} \hat{x}_{j, q} \rho_{q} \sigma_{i, q} \sigma_{j, q}
$$

but exploiting the relation $\hat{x}_{i, q} \sigma_{i, q}=c(\lambda)=c, \forall i=1, \ldots,(h-1)$ and $\hat{x}_{i, q}=1, \forall i=h, \ldots, n_{q}$, it is tedious but straightforward to see that:

$$
V_{q}=\sum_{i=1}^{h-1} c^{2}+c^{2} \rho_{q} \sum_{i=1}^{h-1} \sum_{j \neq i} 1+\sum_{i=h}^{n_{q}} \sigma_{i, q}^{2}+2 \rho_{q} \sum_{i=h}^{n_{q}} \sigma_{i, q} \sum_{j>i} \sigma_{j, q}+\sum_{j=1}^{h-1} c \rho_{q} \sum_{i=h}^{n_{q}} \sigma_{i, q}+\sum_{i=1}^{h-1} c \rho_{q} \sum_{j=h}^{n_{q}} \sigma_{j, q}
$$


Then, manipulating the above expression and writing the expression as a second order polynomial in the variable $c(\lambda)$, we obtain:

$$
V_{q}=c^{2}\left[(h-1)+\rho_{q}(h-1)(h-2)\right]+c 2(h-1) \rho_{q} \sum_{i=h}^{n_{q}} \sigma_{i, q}+2 \rho_{q} \sum_{i=h}^{n_{q}} \sigma_{i, q} \sum_{j>i} \sigma_{j, q}+\sum_{i=h}^{n_{q}} \sigma_{i, q}^{2}
$$

which gives the overall variance, of the $q$-th group, in the subinterval where $(h-1)$ policies are active. Now, by recalling that there (see also (14a)):

$$
\hat{x}_{i, q}(\lambda) \sigma_{i, q}=c(\lambda)=\frac{\lambda}{a_{q}\left[1+\rho_{q}(h-2)\right]}-\rho_{q} \frac{\sum_{j=h}^{n_{q}} \sigma_{j, q}}{\left[1+\rho_{q}(h-2)\right]}
$$

we are able to rewrite the formulation of the variance emphasizing the dependence from the shadow price $\lambda$. In particular, we may write:

$$
V_{q}(\lambda)=\alpha_{q, h}^{\prime} \lambda^{2}+\beta_{q, h}^{\prime} \lambda+\gamma_{q, h}
$$

where, specifically (see Appendix A):

$$
\begin{aligned}
\alpha_{q, h}^{\prime} & =\frac{(h-1)}{a_{q}^{2}\left[1+\rho_{q}(h-2)\right]} \\
\beta_{q, h}^{\prime} & =0 \\
\gamma_{q, h}^{\prime} & =-\frac{\rho_{q}^{2}(h-1)\left(\sum_{i=h}^{n_{q}} \sigma_{i, q}\right)^{2}}{1+\rho_{q}(h-2)}+2 \rho_{q} \sum_{i=h}^{n_{q}} \sigma_{i, q} \sum_{j=h+1}^{n_{q}} \sigma_{j, q}+\sum_{i=h}^{n_{q}} \sigma_{i, q}^{2}
\end{aligned}
$$

noting also that $\alpha_{q, h}^{\prime}=\alpha_{q, h}$ given in the (19).

Now, let us evaluate the global variance of the entire portfolio, $V(\lambda)$, coming from all groups $g$. As before, in any subinterval of $\lambda$, where each group has a group specific number $(h-1)_{q}$ of active policies, the global variance is given simply by the sum of the variances of each of them, owing to the null correlation between groups.

$$
V(\lambda)=\lambda^{2} \sum_{q=1}^{g} \alpha_{q, h}+\sum_{q=1}^{g} \gamma_{q, h}=\lambda^{2} \alpha_{h}+\gamma_{h}
$$

We underline that the graph of the variance is an union of parabolas, whose symmetry axis is, for each of them, the vertical axis $(\lambda=0)$ with concavity coefficient positive and increasing with $h$ (recall that such a coefficient is also the slope of the straight line of the corresponding expectation), while the role of $\gamma_{h}$ is to grant continuity at the connection points; also the continuity of $V(\lambda)$ comes immediately from the continuity of $\hat{x}_{i, q}(\lambda)$.

Finally, exploiting (25), it is possible to write the relation which links, in any subinterval, the global expected return and the global variance, through a function $V(E)$. It is:

$$
V(E)=\frac{\left(E-\beta_{h}\right)^{2}}{\alpha_{h}^{2}} \alpha_{h}+\gamma_{h}=\frac{\left(E-\beta_{h}\right)^{2}}{\alpha_{h}}+\gamma_{h}
$$

This way, in every single subinterval, we are able to write $V$ as a quadratic function of $E$.

Overall the intervals of the efficient set the graph of $V(E)$ is therefore an union of parabolas. It turns out that, also at the connection points, $V(E)$ is a continuous and differentiable function (without kinks). The differentiability is intuitively explained considering $\frac{1}{2} \frac{\partial V}{\partial E}=\lambda$ and recalling the continuity of $\lambda$ along the optimum path and, specifically, Corollary 1 and Property 5. Of course, continuity is implied by differentiability. For a formal proof of continuity and differentiability of $V(E)$, see Appendix B. 


\section{Comparison with the case of no correlation}

Without any claim to use realistic data but in order to appreciate the role of correlation, we provide a picture of some efficient sets in the mean-variance space for a hypothetical portfolio of policies under different group correlation structures.

Then we consider a test portfolio of fifty policies (see Table 1) divided in 5 groups characterized by a given structure of the ratios standard deviation-expectation, to be seen as the index $a_{q}$ of relative riskiness. To this portfolio we apply three different group correlation structures with equally spaced correlation coefficients going from 0.05 to 0.25 ; precisely, in the first case (direct) the ranking of the correlation is the same of the riskiness; in the second (inverse) case, the ranking is the opposite and, in the third (unsystematic) one, there is no systematic association between the rankings. All three scenarios have been compared with the case of zero correlation within all groups.

As expected (see Figure 4), the first case with direct relation between riskiness and correlation (dot curve) reveals the highest variance for any level of mean, followed by the no systematic association case and the inverse relation case. In the no correlation case, the variance is remarkably lower than under group correlation, especially for the highest level of the expected gain.

In conclusion, proportional reinsurance may be quite helpful to keep under control the variance of a portfolio especially under (group) correlation.

\begin{tabular}{|r|r|r|r|r|r|}
\hline$q$ & $\mathbf{1}$ & $\mathbf{2}$ & $\mathbf{3}$ & $\mathbf{4}$ & $\mathbf{5}$ \\
\hline \hline$a_{q}$ & 2.650 & 2.955 & 3.156 & 4.013 & 4.891 \\
\hline \hline$i$ & \multicolumn{5}{|c|}{ Expected Return $m_{i, q}$} \\
\hline \hline $\mathbf{1}$ & 55 & 86 & 34 & 205 & 101 \\
$\mathbf{2}$ & 49 & 75 & 29 & 180 & 91 \\
$\mathbf{3}$ & 35 & 69 & 24 & 155 & 79 \\
$\mathbf{4}$ & 31 & 58 & 20 & 147 & 62 \\
$\mathbf{5}$ & 30 & 55 & 19 & 137 & 59 \\
$\mathbf{6}$ & 28 & 46 & 17 & 121 & 50 \\
$\mathbf{7}$ & 23 & 39 & 14 & 101 & 42 \\
$\mathbf{8}$ & 20 & 32 & 11 & 93 & 39 \\
$\mathbf{9}$ & 15 & 27 & 9 & 80 & 26 \\
$\mathbf{1 0}$ & 13 & 20 & 7 & 67 & 17 \\
\hline \hline$i$ & \multicolumn{5}{|c|}{ Standard Deviation $\sigma_{i, q}$} \\
\hline \hline $\mathbf{1}$ & 145,76 & 254,12 & 107,30 & 822,74 & 494,02 \\
$\mathbf{2}$ & 129,86 & 221,62 & 91,52 & 722,41 & 445,11 \\
$\mathbf{3}$ & 92,76 & 203,89 & 75,74 & 622,07 & 386,41 \\
$\mathbf{4}$ & 82,16 & 171,38 & 63,12 & 589,97 & 303,26 \\
$\mathbf{5}$ & 79,51 & 162,52 & 59,96 & 549,83 & 288,59 \\
$\mathbf{6}$ & 74,20 & 135,93 & 53,65 & 485,62 & 244,57 \\
$\mathbf{7}$ & 60,95 & 115,24 & 44,18 & 405,35 & 205,43 \\
$\mathbf{8}$ & 53,00 & 94,56 & 34,71 & 373,24 & 190,76 \\
$\mathbf{9}$ & 39,75 & 79,78 & 28,40 & 321,07 & 127,17 \\
$\mathbf{1 0}$ & 34,45 & 59,10 & 22,09 & 268,90 & 83,15 \\
\hline
\end{tabular}

Table 1: Test portfolio. 


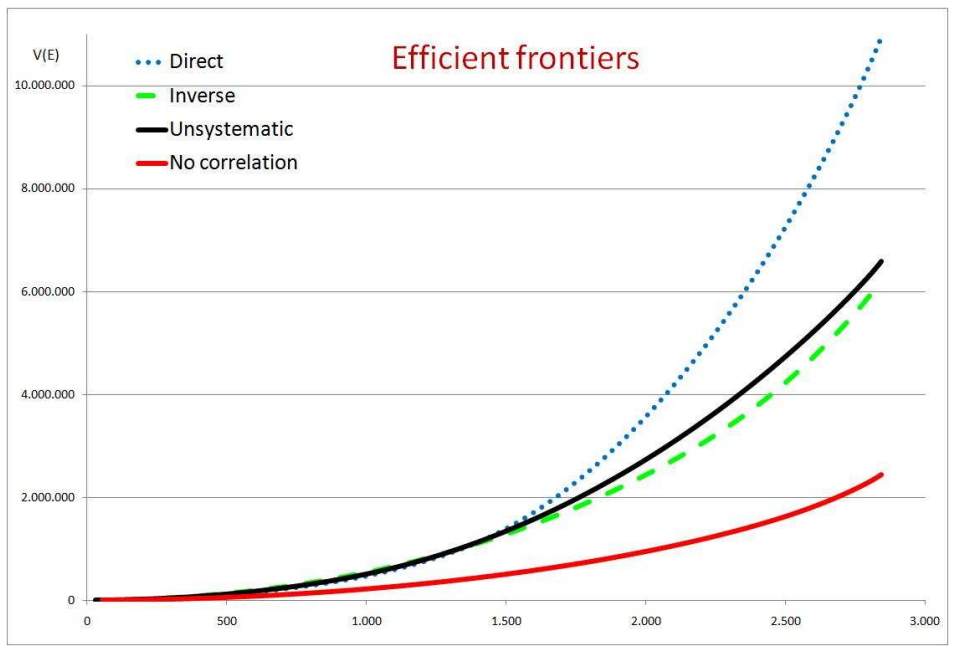

Figure 4: An example of mean-variance efficient frontiers.

\section{Conclusions}

We analyzed in this paper the problem of finding a closed form mean-variance efficient solution to the so called group correlation problem in proportional reinsurance. This problem was originally raised by de Finetti in his path-breaking paper [9]; there, he gave a quick hint to some key properties of the solution but without giving closed form formulae. A subsequent treatment of this issue may be found in a paper by Gigante [16]; she was able to apply the Karush-Kuhn-Tucker conditions to the constrained quadratic optimization problem arising from the mean-variance approach to proportional reinsurance. This way she showed that, under group correlation, a fast version of the critical line algorithm could be applied to obtain rather quickly the whole mean-variance set of efficient retentions. Anyway, she did not get closed form formulae.

In this paper we fill this gap and offer a complete closed form solution of the proportional reinsurance problem under group correlation, jointly with an insightful interpretation of the economic meaning of the solution set. To explain our results we make large recourse to de Finetti's original approach, based on the so called advantage functions technique.

More precisely, we show that there is a one-to-one correspondence between the set of efficient mean-variance retentions and the closed interval of values of a shadow price parameter; we offer closed form formulae of these vectors as a function of the shadow price; we show that in the $n$-dimensional space of retentions, the meanvariance efficient set is piecewise linear and continuous at corner points; we specify the values of the shadow price and hence of the retention vector corresponding to the corner points of the efficient set; we show that the geometric picture of the efficient retention set in a mean-variance space is a, piecewise continuous and without kinks, union of parabolas, whose equations are explicitly derived. In our opinion these results may be useful for the sake of comparison between results coming from the traditional mean-variance approach and those coming from modern mean-risk alternative models. 


\section{Appendix A}

Computation of variance of a generic group.

Recalling (28) it is:

$$
c^{2}(\lambda)=\frac{\lambda^{2}}{a_{q}^{2}\left[1+\rho_{q}(h-2)\right]^{2}}-2 \rho_{q} \lambda \frac{\sum_{j=h}^{n_{q}} \sigma_{j, q}}{a_{q}\left[1+\rho_{q}(h-2)\right]^{2}}+\rho_{q}^{2} \frac{\left(\sum_{j=h}^{n_{q}} \sigma_{j, q}\right)^{2}}{\left[1+\rho_{q}(h-2)\right]^{2}}
$$

Then, inserting $c(\lambda)$ and $c^{2}(\lambda)$ in(27) we may opportunely write:

$$
\begin{aligned}
V_{q}(\lambda) & =(h-1)\left[1+\rho_{q}(h-2)\right]\left[\frac{\lambda^{2}}{a_{q}^{2}\left[1+\rho_{q}(h-2)\right]^{2}}-2 \rho_{q} \lambda \frac{\sum_{j=h}^{n_{q}} \sigma_{j, q}}{a_{q}\left[1+\rho_{q}(h-2)\right]^{2}}\right]+ \\
& +(h-1)\left[1+\rho_{q}(h-2)\right] \rho_{q}^{2} \frac{\left(\sum_{j=h}^{n_{q}} \sigma_{j, q}\right)^{2}}{\left[1+\rho_{q}(h-2)\right]^{2}}+ \\
& +2(h-1) \rho_{q} \sum_{i=h}^{n_{q}} \sigma_{i, q}\left[\frac{\lambda}{a_{q}\left[1+\rho_{q}(h-2)\right]}-\rho_{q} \frac{\sum_{i=h}^{n_{q}} \sigma_{i, q}}{\left[1+\rho_{q}(h-2)\right]}\right]+2 \rho_{q} \sum_{i=h}^{n_{q}} \sigma_{i, q} \sum_{j>i} \sigma_{j, q}+\sum_{i=h}^{n_{q}} \sigma_{i, q}^{2}
\end{aligned}
$$

which may bee conveniently rewritten in the form

$$
\begin{aligned}
V_{q}(\lambda) & =\left[\frac{(h-1)\left[1+\rho_{q}(h-2)\right]}{a_{q}^{2}\left[1+\rho_{q}(h-2)\right]^{2}}\right] \lambda^{2}- \\
& -2 \rho_{q}(h-1) \sum_{j=h}^{n_{q}} \sigma_{j, q}\left[\frac{\left[1+\rho_{q}(h-2)\right]}{a_{q}\left[1+\rho_{q}(h-2)\right]^{2}}\right] \lambda+2 \rho_{q}(h-1) \sum_{j=h}^{n_{q}} \sigma_{j, q}\left[\frac{1}{a_{q}\left[1+\rho_{q}(h-2)\right]}\right] \lambda+ \\
& +(h-1) \rho_{q}^{2} \frac{\left(\sum_{j=h}^{n_{q}} \sigma_{j, q}\right)^{2}}{\left[1+\rho_{q}(h-2)\right]}-2(h-1) \rho_{q}^{2} \frac{\sum_{i=h}^{n_{q}} \sigma_{i, q} \sum_{i=h}^{n_{q}} \sigma_{i, q}}{\left[1+\rho_{q}(h-2)\right]}+2 \rho_{q} \sum_{i=h}^{n_{q}} \sigma_{i, q} \sum_{i+1}^{n_{q}} \sigma_{i, q}+\sum_{i=h}^{n_{q}} \sigma_{i, q}^{2}
\end{aligned}
$$

and finally:

$$
V_{q}(\lambda)=\frac{(h-1)}{a_{q}^{2}\left[1+\rho_{q}(h-2)\right]} \lambda^{2}-(h-1) \rho_{q}^{2} \frac{\sum_{i=h}^{n_{q}} \sigma_{i, q} \sum_{i=h}^{n_{q}} \sigma_{i, q}}{\left[1+\rho_{q}(h-2)\right]}+2 \rho_{q} \sum_{i=h}^{n_{q}} \sigma_{i, q} \sum_{i+1}^{n_{q}} \sigma_{i, q}+\sum_{i=h}^{n_{q}} \sigma_{i, q}^{2}
$$

so that:

$$
V_{q}(\lambda)=\alpha_{q, h}^{\prime} \lambda^{2}+\beta_{q, h}^{\prime} \lambda+\gamma_{q, h}^{\prime}
$$

with:

$$
\begin{aligned}
\alpha_{q, h}^{\prime} & =\frac{(h-1)}{a_{q}^{2}\left[1+\rho_{q}(h-2)\right]} \\
\beta_{q, h}^{\prime} & =0 \\
\gamma_{q, h}^{\prime} & =-\frac{\rho_{q}^{2}(h-1)\left(\sum_{i=h}^{n_{q}} \sigma_{i, q}\right)^{2}}{1+\rho_{q}(h-2)}+2 \rho_{q} \sum_{i=h}^{n_{q}} \sigma_{i, q} \sum_{j=h+1}^{n_{q}} \sigma_{j, q}+\sum_{i=h}^{n_{q}} \sigma_{i, q}^{2}
\end{aligned}
$$




\section{Appendix B}

Proof of continuity and differentiability of $V(E)$.

Here we apply general results of duality theory to the reinsurance problem to characterize some relationships. Let us restate here our problem

$$
\begin{aligned}
v(E)=\min & f(\mathbf{x}) \\
& \mathbf{m}^{\top} \mathbf{x} \geq E \\
& \mathbf{x} \in X
\end{aligned}
$$

where $X:=\left\{\mathbf{x} \in R^{n}: \mathbf{0} \leq \mathbf{x} \leq \mathbf{1}\right\}, f(x):=(1 / 2) \mathbf{x}^{\top} C \mathbf{x}$. Viewing (43) as an instance of a non-linear optimization problem, we note that the objective function $f(\mathbf{x})$ is strictly convex, the explicit constraint $-\mathbf{m}^{\top} \mathbf{x} \leq-E$ is convex (in fact affine), and the implicit constraint $\mathbf{x} \in X$ refers to a convex set $X$. Moreover the Slater's constraint qualification is satisfied for $E<\mathbf{m}^{\top} \mathbf{1}$, i.e., there exists a feasible point $\overline{\mathbf{x}}=\mathbf{1} \in X$ such that $\mathbf{m}^{\top} \overline{\mathbf{x}}>E$.

Let the dual of (43) be defined through the Lagrangian function

$$
L(\lambda, E):=\min _{\mathbf{x} \in X} f(\mathbf{x})+\lambda\left(E-\mathbf{m}^{\top} \mathbf{x}\right)=\lambda E+\min _{\mathbf{x} \in X} f(\mathbf{x})-\lambda \mathbf{m}^{\top} \mathbf{x}
$$

Let

$$
\bar{L}(\lambda):=\min _{\mathbf{x} \in X} f(\mathbf{x})-\lambda \mathbf{m}^{\top} \mathbf{x}
$$

so that

$$
L(\lambda, E)=\lambda E+\bar{L}(\lambda)
$$

then the dual problem of (43) is

$$
d(E):=\max _{\lambda \geq 0} L(\lambda, E)=\max _{\lambda \geq 0} \lambda E+\bar{L}(\lambda)
$$

Under the stated conditions we know that strong duality holds, i.e., for each $E$ there exist an optimal $\hat{\mathbf{x}}$ for (43) and an optimal $\hat{\lambda}$ for (44), obviously depending on $E$, such that,

$$
d(E)=v(E)=\bar{L}(\hat{\lambda}, E)=\hat{\lambda} E+f(\hat{\mathbf{x}})-\hat{\lambda} \mathbf{m}^{\top} \hat{\mathbf{x}}=f(\hat{\mathbf{x}})
$$

Note that (45) implies the complementarity condition $\hat{\lambda}\left(\mathbf{m}^{\top} \hat{\mathbf{x}}-E\right)=0$. Now we have that $v(E)$ is an increasing function from $(43)$ and $d(E)$ is convex from (44), being the pointwise maximum of affine functions in $E$. Then by $(45)$ and recalling also that $v(E)=1 / 2 V(E)$, we have that $V(E)$ is a monotonically increasing convex function.

In general $V(E)$ is not everywhere differentiable. Examples can be given where $V(E)$ exhibits kink points.

In our case differentiability can be proven by a direct argument based on the previous results as follows. The left derivative of the variance is immediately obtained just by computing the first derivative of the expression (34):

$$
V^{\prime}\left(E_{h}\right)=2 \frac{\left(E_{h}-\beta_{h}\right)}{\alpha_{h}}
$$

and inserting:

$$
E_{h}=\alpha_{h} \lambda_{h}+\beta_{h}
$$

we obtain:

$$
V^{\prime}\left(E_{h}\right)=2 \lambda_{h}
$$

As for the right derivative, we must compute the limit:

$$
\lim _{E \rightarrow E_{h}^{+}} V^{\prime}(E)
$$


In a right neighborhood of $E_{h}$ :

$$
V^{\prime}(E)=2 \frac{\left(E-\beta_{h}\right)}{\alpha_{h}}
$$

and substituting:

$$
\begin{gathered}
E=\alpha_{h-1} \lambda+\beta_{h-1} \\
V^{\prime}(E)=2 \lambda
\end{gathered}
$$

Now, when $E \rightarrow E_{h}^{+}, \lambda \rightarrow \lambda_{h}^{+}$and so

$$
\lim _{E \rightarrow E_{h}^{+}} V^{\prime}(E)=\lim _{\lambda \rightarrow \lambda_{h}^{+}}=2 \lambda_{h}
$$




\section{References}

[1] Artzner, P., F. Delbaen, J.M. Eber and D. Heath (1999), Coherent measures of risk, Mathematical Finance, 9, 203-228

[2] Balbás, A., B. Balbás and A. Heras (2009), Optimal reinsurance with general risk measures, Insurance: Mathematics and Economics, 44, 374-384

[3] Barone, L. (2008), Bruno de Finetti and the case of the critical line's last segment, Insurance: Mathematics and Economics, 42-1, 359-377

[4] Berliner, B. (1977), A risk measure alternative to the variance, The ASTIN Bullettin, 9, 42-58

[5] Borch, K. (1974), The mathematical theory of insurance, Lexington Books

[6] Bühlmann, H. (1970), Mathematical methods in risk theory, Springer

[7] Bühlmann, H. (2006), Encounters with ideas of Bruno De Finetti, presented at the Sixth Scientific Conference on Insurance and Finance of the German Association for Actuarial and Financial Mathematics, Berlin

[8] Bühlmann, H. and H. Gerber (1978), Risk bearing and the reinsurance market, The ASTIN Bullettin, 10, $12-24$

[9] de Finetti, B. (1940), Il problema dei pieni, Giornale Istituto Italiano Attuari, 9, 1-88; English translation by L. Barone available as "The problem of Full-risk insurances", Ch. 1 'The problem in a single accounting period', Journal of Investment Management, 4, 19-43, 2006

[10] de Finetti, B. (1942), Impostazione individuale e impostazione collettiva del problema della riassicurazione, Giornale Istituto Italiano Attuari, 13, 28-33;

[11] de Finetti, B. (1957), Su una impostazione alternativa della teoria collettiva del rischio, Transaction XVth International Congress of Actuaries, Mallon, New York, 2, 433-443;

[12] Dickson, D.C.M. and H.R. Waters (1997), Relative Reinsurance retention levels, The ASTIN Bullettin, 27-2, 207-227

[13] Gerber, H.U. (1984), Equilibria in a proportional reinsurance market, Insurance, Mathematics and Economics 3, 97-100

[14] Gerber, H.U. (1974), On additive premium calculation principles, The ASTIN Bullettin, 7-3, 215-222

[15] Gerber, H. U. and E.S W. Shiu (2003), Economic Ideas of Bruno De Finetti in the Wiener Process Model, Metodi Statistici per la Finanza e le Assicurazioni, 75-95

[16] Gigante, P. (1990), L'approccio di B. de Finetti per la riassicurazione individuale proporzionale. Modelli semplificati e considerazioni operative, Rivista di Matematica per le Scienze Economiche e Sociali, 13, $1^{\circ}-2^{\circ}, 147-161$

[17] Glineur, F. and J.F. Walhin (2006), de Finetti's retention problem for proportional reinsurance revisited, German Actuarial Bulletin, 28-1, 451-462

[18] Goovaerts, M., R. Kaas, J. Dhaene, and Q. Tang (2004), Some new classes of consistent risk measures, Insurance: Mathematics and Economics, 34, 505-516 
[19] Karush, W. (1939), Minima of functions of several variables with inequalities as side constraints, M.Sc. dissertation, Department of Mathematics, University of Chicago, Chicago, IL, USA

[20] Kuhn, H.W. and A.W. Tucker (1951), Nonlinear programming, Proceedings of the Second Berkeley Symposium on Mathematical Statistics and Probability, J. Neyman ed., University of California Press, Berkeley, CA, USA

[21] Lampaert, I. and J.F. Walhin (2005), On the optimality of proportional reinsurance, Scandinavian Actuarial Journal, 3, 225-239

[22] Landsman, Z. and M. Sherris (2001), Risk measures and insurance premium principles, Insurance: Mathematics and Economics, 29, 103-115

[23] Markowitz, H. (1952), Portfolio selection, Journal of Finance, 6, 77-91

[24] Markowitz, H. (1956), The optimization of quadratic functions subject to linear constraints, Naval Research Logistics Quarterly, 3, 111-133

[25] Markowitz, H. (1959), Portfolio selection: efficient diversification of investments. New York: John Wiley \& Sons. (reprinted by Yale University Press, 1970; 2nd ed. Basil Blackwell, 1991)

[26] Markowitz, H. (2006), de Finetti scoops Markowitz, Journal of Investment Management, 4, 5-18

[27] Miller, N. and A. Ruszczynski (2008), Risk-adjusted probability measures in portfolio optimization with coherent measures of risk, European Journal of Operational Research, 191, 193-206

[28] Pressacco, F. (1986), Separation theorems in proportional reinsurance, Insurance and Risk Theory, M. Goovaerts et al. eds., Reidel Publishing, 209-215

[29] Pressacco, F. and P. Serafini (2007), The origins of the mean-variance approach in finance: revisiting de Finetti 65 years later, Decisions in Economics and Finance, 10-1, 19-49

[30] Rockafellar, R.T., S. Uryasev and M. Zabarankin (2006), Generalized deviations in risk analysis, Finance \& Stochastics, 10, 51-74 